\title{
High levels of low back disability, but not low back pain intensity, are associated with reduced physical activity: a community-based, cross-sectional study
}

Bothaina Alyousef ( $\sim$ Bothaina.Alyousef@monash.edu )

Monash University https://orcid.org/0000-0003-3944-4576

Zeinab Kazemi

Tehran University of Medical Sciences

Flavia M. Cicuttini

Monash University Faculty of Medicine Nursing and Health Sciences

Stephane Heritier

Monash University Faculty of Medicine Nursing and Health Sciences

Yuanyuan Wang

Monash University Faculty of Medicine Nursing and Health Sciences

Donna M. Urquhart

Monash University Faculty of Medicine Nursing and Health Sciences

Research article

Keywords: low back pain, back disability, pain intensity, physical activity, community-based

Posted Date: September 21st, 2020

DOl: https://doi.org/10.21203/rs.3.rs-74076/v1

License: (9) (i) This work is licensed under a Creative Commons Attribution 4.0 International License.

Read Full License 


\section{Abstract \\ Background}

Although clinical guidelines recommend physical activity for low back pain (LBP), our understanding of the physical activity undertaken by people with LBP is limited. We examined the amounts, intensities and types of physical activity performed by community-based women with different levels of pain and disability.

\section{Methods}

542 women were invited to participate. Physical activity was measured using the International Physical Activity Questionnaire, and LBP and disability were assessed using the Chronic Pain Grade Questionnaire. Participants were categorised into no/low or high pain intensity and disability groups.

\section{Results}

Women with high levels of back disability performed half the total physical activity of those with no/low levels of back disability (MET(hours/week): median $(95 \% \mathrm{Cl})=27.3(13.2-41.4)$ vs. $53.9(44.9-62.8), p=$ $0.002)$, including less moderate $(17.5(10.4-24.7)$ vs. $32.1(26.8-37.4), p<0.001)$ and domestic and gardening activity $(14.4(7.2-21.7)$ vs. $23.5(19.0-28.0), p=0.02)$. Moreover, fewer women with high disability participated in vigorous $(3(8.11 \%)$ vs. $134(32.0 \%), \mathrm{OR}(95 \% \mathrm{Cl})=0.17(0.04-0.75), \mathrm{p}=0.02)$ and leisure activities $(17(45.9 \%)$ vs. $294(70.2 \%), 0 R(95 \% \mathrm{Cl})=0.44(0.21-0.94), p=0.03)$ compared to those with no/low disability. There were no differences in physical activity between women with no/low and high pain intensity (all $p>0.05$ ).

\section{Conclusion}

High levels of back disability, but not back pain intensity, were associated with reduced physical activity, including less total activity, moderate and vigorous intense activity, and discretionary activity. Further work is needed to determine whether targeting these types and intensities of physical activity will help in improving physical activity levels and reducing back disability.

\section{Introduction}

Low back pain (LBP) is the leading cause of disability globally, with years lived with disability exceeding that of diabetes and cardiovascular disease (1). Despite the increased societal and financial burden of LBP (2), effective treatments are limited. National and international clinical guidelines consistently recommend physical activity for the management of acute and chronic LBP (3). However, the condition of LBP itself, including the associated back disability, may significantly limit the physical activity performed 
by individuals, and in turn their involvement in family, work and social activities. This may lead to complex social and health issues, such as social isolation, psychological issues, including depression and/or anxiety, and addiction to prescribed medications, such as opioids (4).

A number of systematic reviews have examined the relationship between physical activity and LBP and disability, with a focus on determining whether reduced physical activity is a risk factor for the development and progression of $\operatorname{LBP}(5,6)$. In contrast, few studies have investigated the physical activity patterns of individuals with LBP. While a systematic review found individuals with high levels of low back disability performed less physical activity (7), the review did not examine the specific amounts, types and intensities of physical activity performed. Moreover, recent cross-sectional studies found conflicting results when examining the association between physical activity and LBP and disability (8, 9). While Sokunbi et al (2016) found no association between pain and disability and the amount of physical activity performed by nurses with chronic LBP(9), Ezeukwu et al (2019) found low levels of physical activity in patients presenting to a physiotherapy clinic with chronic, non-specific LBP and disability (8). Thus, we are lacking a comprehensive understanding of the amount and nature of physical activity performed by community-based individuals with LBP and disability.

This study aimed to examine the amounts, intensities and types of physical activity performed by community-based individuals with different levels of LBP intensity and disability. We hypothesised that individuals with high levels of pain and disability would perform less physical activity of all types and intensities. An understanding of the physical activity patterns of individuals with LBP has the potential to provide important information about the burden of LBP and disability, the particular subgroups of individuals with LBP that perform reduced physical activity, as well as the types and intensities of physical activity that may be important to target in improving physical activity and reducing low back disability.

\section{Methods}

\section{Study sample}

Participants were recruited from a parent study of 1,423 community-dwelling women aged between 18 and 75 years. The sample was randomly recruited from a database that was derived from the Victorian Electoral Roll from 2002 to 2003. Details of the parent study methodology has been previously reported (10). Of the 1423 participants, 754 were willing to be contacted to participate in further research and 542 consented to take part in this study upon re-contact. Written informed consent was obtained from all participants and the research was approved by Monash University Human Research and Ethics Committee (CF07/2961-2006000113).

\section{Demographics}

Age, weight and height were self-reported by participants and body mass index (BMl; $\left.\mathrm{kg} / \mathrm{m}^{2}\right)$ was calculated. Participants were considered working if they had paid jobs, or were involved in volunteer work, 
course work and unpaid work outside the home. Depression was assessed based on the following question "Have you been diagnosed with depression in the past 2 years?".

\section{Low back pain intensity and disability}

The Chronic Pain Grade (CPG) questionnaire was used to assess LBP intensity and disability. It is a selfadministrated instrument that has been shown to be valid and reliable to assess chronic pain in community-based research (11). It consists of seven items that measure back pain intensity and degree of disability. Each item has an 11-point Likert scale that is summed to give a score ranging from 0 to 100 . High levels of pain intensity and disability were defined as scores equal to or more than 50 and no or low pain intensity and disability were defined as scores less than 50 according to the CPG (11).

\section{Physical activity}

The long version of the International Physical Activity Questionnaire (IPAQ) was used to assess physical activity. The IPAQ consists of 31 items, which capture different domains of physical activity over the last 7 days and include physical activity associated with leisure-time, work, active transportation, and domestic and garden activities. Physical activity was also categorised according to intensity as vigorous, moderate and walking activities (Table 1). Each physical activity domain was reported in terms of the number of days and hours/minutes per day that the activity was performed in the past 7 days. The sum of hours per week for each activity was weighed to an assigned energy expenditure equivalent, the metabolic equivalent task (MET), based on the intensity of this physical activity domain (MET $x$ hours per day $x$ number of days per week) $(12,13)$. Based on the IPAQ guidelines, any participant reporting over 16 hours of physical activity per day or more than 7 days a week for activity was considered an outlier and omitted from the analysis(14).

Table 1

Intensity of physical activity as specified by the International Physical Activity Questionnaire domains.

Intensity of Activity specific items
physical
activity

\begin{tabular}{|ll|}
\hline Vigorous & Includes vigorous work-related activity and vigorous leisure activity \\
\hline Moderate & $\begin{array}{l}\text { Includes moderate work-related activity, vigorous and moderate domestic and garden } \\
\text { activities, moderate leisure activity and cycling transport (with the exclusion of } \\
\text { walking) }\end{array}$ \\
\hline Walking & Includes walking at work, and for transport and leisure \\
\hline
\end{tabular}

\section{Statistical analysis}

Descriptive statistics for age, BMI, depression, employment status and physical activity domains were tabulated based on LBP and disability groups, and differences between the groups were compared using chi-square test $\left(\chi^{2}\right)$ for categorical variables, Mann-Whitney $U$ test for non-gaussian continuous variables, and independent samples t-test for normally distributed, continuous variables. 
To determine the association between back pain and disability and the physical activity domains of domestic and garden activity, walking, moderate physical activity and total physical activity (MET, hours/week), estimated adjusted medians were obtained using quantile regression analysis and the $95 \%$ confidence intervals $(\mathrm{Cl})$ were obtained by bootstrapping with 1000 replicates from the quantile regression. There was insufficient participation in work-related activity, active transportation, vigorous and leisure physical activities and thus these activities were dichotomised as individuals participating or not. Binary logistic regression was used to examine the association between LBP and disability and these physical activity domains.

With regards to the data for sitting time, the discreteness of the data made quantile regression unreliable as a continuous endpoint distribution is required for this technique to be valid. We adopted a square root transformation of the sitting time endpoint to achieve approximate normality (up to rounding). Linear regression was then used to examine the association between LBP and disability and the transformed endpoint; the estimated marginal medians and $95 \%$ Cls were obtained by back-transformation of the adjusted means.

Multivariable analyses were adjusted for potential confounders in model 1; age, BMI and depression, in model 2 we added working status as it particularly showed in the univariate analysis that it was significantly different between women with high levels of pain intensity and disability and those with no or low levels of pain intensity and disability. Statistical significance was denoted by a $p$ value of less than 0.05. Data were analysed using Stata 12.0 SE (StataCorp).

\section{Results}

Of the 542 participants who were recruited for this study, 506 (93.4\%) returned the study questionnaire. 463 participants were included in the analyses after excluding 43 , as they were considered outliers (as per the IPAQ guidelines) (14). However, there were no differences between the outliers and those included in the final analyses in terms of age, BMI, depression, pain intensity and disability $(p=0.07-0.63)$, with the exception of a higher percentage of women employed in the outlier group (39 (90.7\%) vs. $278(61.2 \%), p<$ 0.001).

The mean (SD) age and BMI of the study cohort was 56.9 (12.7) years and $27.4(5.6) \mathrm{kg} / \mathrm{m}^{2}$, respectively. A total of $278(60.3 \%)$ participants were employed and 62 (13.4\%) self-reported depression. The mean (SD) LBP intensity and disability scores were 25.6 (22.3) and 13.9 (20.5) respectively. The median (interquartile range (IQR)) total physical activity (MET, hours/week) for the cohort was 49.1 (82.1).

The characteristics of participants with no/low and high LBP intensity are presented in Table 2. High intensity back pain was reported in $71(15.3 \%)$ women. Compared to women with no or low intensity back pain, those with high intensity back pain had a greater BMI (mean(SD) = 29.5 (5.3) vs. 27.0 (5.7), p = $0.001)$, were more likely to be depressed $(16(22.5 \%)$ vs. $46(11.7 \%), p=0.01)$ and less likely to be employed (33 $(47.1 \%)$ vs. $245(62.6 \%), p=0.02)$. There were no differences between the pain intensity 
groups in their physical activity and sitting time, with the exception that fewer women with high pain intensity reported doing physical activity at work (16 (23.5\%) vs. $142(36.6 \%), p=0.04)$. 
Table 2

Characteristics of participants with no or low and high levels of low back pain and disability.

\begin{tabular}{|c|c|c|c|c|c|c|c|}
\hline & $\begin{array}{l}\text { Total } \\
\text { cohort } \\
(n= \\
463)^{a}\end{array}$ & $\begin{array}{l}\text { No/low } \\
\text { pain } \\
\text { intensity } \\
(n=392)\end{array}$ & $\begin{array}{l}\text { High } \\
\text { pain } \\
\text { intensity } \\
(n=71)\end{array}$ & $\begin{array}{l}P \\
\text { value }\end{array}$ & $\begin{array}{l}\text { No/ low } \\
\text { disability } \\
(n=425)\end{array}$ & $\begin{array}{l}\text { High } \\
\text { disability } \\
(n=38)\end{array}$ & $\begin{array}{l}P \\
\text { value }\end{array}$ \\
\hline Age $\left(\right.$ years) ${ }^{\mathrm{b}}$ & $\begin{array}{l}56.9 \\
(12.7)\end{array}$ & $\begin{array}{l}56.7 \\
(12.7)\end{array}$ & $\begin{array}{l}57.9 \\
(12.7)\end{array}$ & 0.45 & $\begin{array}{l}56.6 \\
(12.8)\end{array}$ & $\begin{array}{l}59.9 \\
(11.7)\end{array}$ & 0.12 \\
\hline $\begin{array}{l}\text { Body mass index } \\
\left(\mathrm{kg} / \mathrm{m}^{2}\right)^{\mathrm{b}}\end{array}$ & $\begin{array}{l}27.4 \\
(5.6)\end{array}$ & $\begin{array}{l}27.0 \\
(5.7)\end{array}$ & $\begin{array}{l}29.5 \\
(5.3)\end{array}$ & 0.001 & $27.2(5.6)$ & $\begin{array}{l}29.8 \\
(5.7)\end{array}$ & 0.01 \\
\hline Depression, n (\%) & $\begin{array}{l}62 \\
(13.4)\end{array}$ & $46(11.7)$ & $\begin{array}{l}16 \\
(22.5)\end{array}$ & 0.01 & $56(13.2)$ & $6(15.8)$ & 0.65 \\
\hline Working, n (\%) & $\begin{array}{l}278 \\
(60.3)\end{array}$ & $\begin{array}{l}245 \\
(62.6)\end{array}$ & $\begin{array}{l}33 \\
(47.1)\end{array}$ & 0.02 & $264(62.4)$ & $14(36.8)$ & 0.002 \\
\hline \multicolumn{8}{|l|}{ Physical activity } \\
\hline \multicolumn{8}{|c|}{ Types of physical activity } \\
\hline \multicolumn{8}{|l|}{ Work-related activity } \\
\hline$n(\%)$ & $\begin{array}{l}158 \\
(34.7)\end{array}$ & $\begin{array}{l}142 \\
(36.6)\end{array}$ & $\begin{array}{l}16 \\
(23.5)\end{array}$ & 0.04 & 149 (35.6) & $9(24.3)$ & 0.17 \\
\hline MET(hours/week) ${ }^{\mathrm{c}, \mathrm{d}}$ & $\begin{array}{l}1.27 \\
(22.6)\end{array}$ & $0(8.0)$ & $0(0)$ & 0.05 & $0(9.4)$ & $0(0)$ & 0.05 \\
\hline \multicolumn{8}{|l|}{ Active transportation } \\
\hline$n(\%)$ & $\begin{array}{l}315 \\
(69.1)\end{array}$ & $\begin{array}{l}264 \\
(68.0)\end{array}$ & $\begin{array}{l}51 \\
(75.0)\end{array}$ & 0.25 & $291(69.5)$ & $24(64.9)$ & 0.56 \\
\hline $\begin{array}{l}\text { MET(hours/week ) } \\
\mathrm{c}, \mathrm{d}\end{array}$ & $\begin{array}{l}3.3 \\
(11.0)\end{array}$ & $\begin{array}{l}3.3 \\
(11.0)\end{array}$ & $\begin{array}{l}4.95 \\
(11.3)\end{array}$ & 0.22 & $3.3(11.6)$ & $1.7(5.5)$ & 0.09 \\
\hline \multicolumn{8}{|c|}{ Domestic and garden activity } \\
\hline n (\%) & $\begin{array}{l}420 \\
(92.1)\end{array}$ & $\begin{array}{l}358 \\
(92.3)\end{array}$ & $\begin{array}{l}62 \\
(91.2)\end{array}$ & 0.76 & 358 (91.9) & $35(94.6)$ & 0.56 \\
\hline$\underset{c, d}{\text { MET(hours/week ) }}$ & $\begin{array}{l}18.9 \\
(42.8)\end{array}$ & $\begin{array}{l}18.6 \\
(41.3)\end{array}$ & $\begin{array}{l}19.1 \\
(60.0)\end{array}$ & 0.64 & $\begin{array}{l}19.5 \\
(42.0)\end{array}$ & $\begin{array}{l}16.3 \\
(52.5)\end{array}$ & 0.70 \\
\hline
\end{tabular}

\footnotetext{
a Based on the IPAQ guidelines, any participant reporting over 16 hours of physical activity per day or more than 7 days a week for activity should be considered as an outlier, therefore a total of $43(8.6 \%)$ participants were excluded from the analysis; ${ }^{b}$ Mean (standard deviation); ${ }^{C} M e d i a n$ (interquartile range); ${ }^{\mathrm{d}} \mathrm{MET}=$ metabolic equivalent task.
} 


\begin{tabular}{|c|c|c|c|c|c|c|c|}
\hline & $\begin{array}{l}\text { Total } \\
\text { cohort } \\
(n= \\
463)^{a}\end{array}$ & $\begin{array}{l}\text { No/low } \\
\text { pain } \\
\text { intensity } \\
(n=392)\end{array}$ & $\begin{array}{l}\text { High } \\
\text { pain } \\
\text { intensity } \\
(n=71)\end{array}$ & $\begin{array}{l}P \\
\text { value }\end{array}$ & $\begin{array}{l}\text { No/ low } \\
\text { disability } \\
(n=425)\end{array}$ & $\begin{array}{l}\text { High } \\
\text { disability } \\
(n=38)\end{array}$ & $\begin{array}{l}P \\
\text { value }\end{array}$ \\
\hline$n(\%)$ & $\begin{array}{l}311 \\
(68.2)\end{array}$ & $\begin{array}{l}268 \\
(69.1)\end{array}$ & $\begin{array}{l}43 \\
(63.2)\end{array}$ & 0.34 & $294(70.2)$ & $17(45.9)$ & 0.002 \\
\hline $\begin{array}{l}\text { MET(hours/week ) } \\
\mathrm{c}, \mathrm{d}\end{array}$ & $\begin{array}{l}6.0 \\
(16.5)\end{array}$ & $\begin{array}{l}6.0 \\
(16.3)\end{array}$ & $\begin{array}{l}5.5 \\
(19.2)\end{array}$ & 0.67 & $6.6(16.6)$ & $0(6.6)$ & 0.003 \\
\hline \multicolumn{8}{|c|}{ Intensity of physical activity } \\
\hline \multicolumn{8}{|l|}{ Vigorous activity } \\
\hline$n(\%)$ & $\begin{array}{l}137 \\
(30.0)\end{array}$ & $\begin{array}{l}120 \\
(30.9)\end{array}$ & $17(25)$ & 0.33 & $134(32.0)$ & $3(8.11)$ & 0.002 \\
\hline $\begin{array}{l}\text { MET(hours/week ) } \\
\mathrm{c}, \mathrm{d}\end{array}$ & $0(8)$ & $0(8)$ & $0(2)$ & 0.28 & $0(8)$ & $0(0)$ & 0.002 \\
\hline \multicolumn{8}{|l|}{ Moderate activity } \\
\hline $\mathrm{n}(\%)$ & $\begin{array}{l}429 \\
(94.1)\end{array}$ & $\begin{array}{l}366 \\
(94.3)\end{array}$ & $\begin{array}{l}63 \\
(92.7)\end{array}$ & 0.59 & $394(94.0)$ & $35(94.6)$ & 0.89 \\
\hline $\begin{array}{l}\text { MET(hours/week ) } \\
\mathrm{c}, \mathrm{d}\end{array}$ & $\begin{array}{l}27.8 \\
(55)\end{array}$ & $\begin{array}{l}27.8 \\
(53.8)\end{array}$ & $\begin{array}{l}27.4 \\
(73.8)\end{array}$ & 0.71 & $29(55.0)$ & $19(48.8)$ & 0.30 \\
\hline \multicolumn{8}{|l|}{ Walking } \\
\hline n (\%) & $\begin{array}{l}396 \\
(86.8)\end{array}$ & $\begin{array}{l}336 \\
(86.6)\end{array}$ & $\begin{array}{l}60 \\
(88.2)\end{array}$ & 0.71 & $366(87.4)$ & $30(81.1)$ & 0.28 \\
\hline $\begin{array}{l}\text { MET(hours/week ) } \\
c, d\end{array}$ & $\begin{array}{l}10.5 \\
(19.8)\end{array}$ & $\begin{array}{l}11.0 \\
(19.3)\end{array}$ & $\begin{array}{l}8.3 \\
(23.4)\end{array}$ & 0.8 & $\begin{array}{l}11.6 \\
(19.8)\end{array}$ & $\begin{array}{l}6.6 \\
(11.6)\end{array}$ & 0.02 \\
\hline \multicolumn{8}{|l|}{ Total activity } \\
\hline$n(\%)$ & $\begin{array}{l}455 \\
(99.8)\end{array}$ & $\begin{array}{l}387 \\
(99.7)\end{array}$ & $68(100)$ & 0.68 & $418(99.8)$ & $37(100)$ & 0.77 \\
\hline $\begin{array}{l}\text { MET(hours/week ) } \\
\mathrm{c,d}\end{array}$ & $\begin{array}{l}49.1 \\
(82.1)\end{array}$ & $\begin{array}{l}49.7 \\
(78.6)\end{array}$ & $\begin{array}{l}39.2 \\
(92.6)\end{array}$ & 0.97 & $\begin{array}{l}50.6 \\
(82.4)\end{array}$ & $\begin{array}{l}26.4 \\
(66.4)\end{array}$ & 0.01 \\
\hline $\begin{array}{l}\text { Sitting time } \\
\text { (hours/week) }{ }^{c, d}\end{array}$ & $35(24)$ & $35(22)$ & $35(33)$ & 0.85 & $35(24)$ & $36(24)$ & 0.37 \\
\hline \multicolumn{8}{|c|}{$\begin{array}{l}\text { a Based on the IPAQ guidelines, any participant reporting over } 16 \text { hours of physical activity per day or } \\
\text { more than } 7 \text { days a week for activity should be considered as an outlier, therefore a total of } 43(8.6 \%) \\
\text { participants were excluded from the analysis; }{ }^{b} \text { Mean (standard deviation); }{ }^{\text {CMedian (interquartile }} \\
\text { range); }{ }^{d} \text { MET = metabolic equivalent task. }\end{array}$} \\
\hline
\end{tabular}


Thirty-eight (8.2\%) women reported high levels of back disability (Table 2). Compared to women with no or low levels of disability, women with high disability had a higher BMI (mean(SD) $=29.8$ (5.7) vs. 27.2 (5.6), $p=0.01)$ and less likely to be employed (14 (36.8\%) vs. $264(62.4 \%), p=0.002)$. Women with high back disability were also less likely to perform leisure activity (17 (45.9\%) vs. $294(70.2 \%), p=0.002)$ and vigorous activity $(3(8.11 \%)$ vs. $134(32 \%), p=0.002)$, and reported less MET (hours/week) in leisure activity (median (IQR) $=0(6.6)$ vs. $6.6(16.6), p=0.003)$, vigorous activity $(0(0)$ vs. $0(8), p=0.002)$, walking (6.6 (11.6) vs. $11.6(19.8), p=0.02)$ and total physical activity $(26.4(66.4)$ vs. $50.6(82.4), p=$ $0.01)$. There were no significant differences in active transportation, domestic and gardening activity, moderate activity and sitting time between women with high and no/low disability.

\section{Associations Between Low Back Pain Intensity And Physical Activity}

After adjustment for age, BMI and depression (Table 3, model 1), there were no significant differences between women with high levels of LBP intensity and those with no/low LBP intensity in any physical activity (MET, hours/week), including domestic and gardening activities (estimated marginal median $(95 \% \mathrm{Cl})=21.8(13.2-30.4)$ vs. $21.4(17.1-25.6), \mathrm{p}=0.92)$, walking $(11.0(7.1-15.0)$ vs. 11.1 (9.4-12.8), p $=0.98)$, moderate activity $(28.8(18.3-39.4)$ vs. $31.4(25.1-37.7), p=0.67)$, total physical activity (45.6 (19.0-72.3) vs. 53.2 (45.1-61.3), $\mathrm{p}=0.59)$, or sitting time (hours/week) (33.6 (29.5-38.2) vs. 34.8 (32.936.6), $p=0.37$ ). With the addition of adjustment for working status (Table 3, model 2; Fig. 1(A) and (B)), these results remained unchanged. 
Table 3

The association between levels of low back pain intensity and disability and physical activity (MET, hours/week).

\begin{tabular}{|c|c|c|c|c|c|c|}
\hline \multirow[t]{3}{*}{ Domain } & \multicolumn{3}{|c|}{ Low back pain intensity } & \multicolumn{3}{|c|}{ Low back disability } \\
\hline & \multicolumn{2}{|c|}{$\begin{array}{l}\text { Estimated marginal } \\
\text { median }(95 \% \mathrm{Cl})^{\mathrm{a}}\end{array}$} & \multirow[t]{2}{*}{$\begin{array}{l}\mathrm{P} \\
\text { value }\end{array}$} & \multicolumn{2}{|c|}{$\begin{array}{l}\text { Estimated marginal median } \\
(95 \% \mathrm{Cl})^{a}\end{array}$} & \multirow[t]{2}{*}{$\begin{array}{l}\mathrm{P} \\
\text { value }\end{array}$} \\
\hline & No/low & High & & No/low & High & \\
\hline \multicolumn{7}{|l|}{ Model $1^{b}$} \\
\hline $\begin{array}{l}\text { Domestic and garden } \\
\text { activity }\end{array}$ & $\begin{array}{l}21.4(17.1- \\
25.6)\end{array}$ & $\begin{array}{l}21.8(13.2- \\
30.4)\end{array}$ & 0.92 & $\begin{array}{l}22.0(17.8- \\
26.2)\end{array}$ & $\begin{array}{l}16.7(8.1- \\
25.4)\end{array}$ & 0.25 \\
\hline Walking & $\begin{array}{l}11.1(9.4- \\
12.8)\end{array}$ & $\begin{array}{l}11.0(7.1- \\
15.0)\end{array}$ & 0.98 & $\begin{array}{l}11.4(9.8- \\
13.0)\end{array}$ & $\begin{array}{l}7.6(2.7- \\
12.5)\end{array}$ & 0.15 \\
\hline Moderate activity & $\begin{array}{l}31.4(25.1- \\
37.7)\end{array}$ & $\begin{array}{l}28.8(18.3- \\
39.4)\end{array}$ & 0.67 & $\begin{array}{l}32.1(26.5- \\
37.8)\end{array}$ & $\begin{array}{l}19.5(11.3- \\
28.8)\end{array}$ & 0.01 \\
\hline $\begin{array}{l}\text { Total physical } \\
\text { activity }\end{array}$ & $\begin{array}{l}53.2(45.1- \\
61.3)\end{array}$ & $\begin{array}{l}45.6(19.0- \\
72.3)\end{array}$ & 0.59 & $\begin{array}{l}54.4(45.3- \\
63.6)\end{array}$ & $\begin{array}{l}28.4(14.5- \\
42.3)\end{array}$ & 0.001 \\
\hline $\begin{array}{l}\text { Sitting time } \\
\text { (hours/week) }\end{array}$ & $\begin{array}{l}34.8(32.9- \\
36.6)\end{array}$ & $\begin{array}{l}33.6(29.5- \\
38.2)\end{array}$ & 0.37 & $\begin{array}{l}34.5(32.7- \\
36.2)\end{array}$ & $\begin{array}{l}37.2(31.1- \\
43.8)\end{array}$ & 0.41 \\
\hline \multicolumn{7}{|l|}{ Model $2^{c}$} \\
\hline $\begin{array}{l}\text { Domestic and garden } \\
\text { activity }\end{array}$ & $\begin{array}{l}23.4(18.5- \\
28.2)\end{array}$ & $\begin{array}{l}21.0(13.3- \\
28.7)\end{array}$ & 0.57 & $\begin{array}{l}23.5(19.0- \\
28.0)\end{array}$ & $\begin{array}{l}14.4(7.2- \\
21.7)\end{array}$ & 0.02 \\
\hline Walking & $\begin{array}{l}11.0(9.2- \\
12.7)\end{array}$ & $\begin{array}{l}12.0(7.0- \\
17.1)\end{array}$ & 0.71 & $\begin{array}{l}11.5(9.8- \\
13.1)^{-}\end{array}$ & $\begin{array}{l}7.4(2.6- \\
12.1)\end{array}$ & 0.12 \\
\hline Moderate activity & $\begin{array}{l}32.4(26.7- \\
38.1)\end{array}$ & $\begin{array}{l}23.2(10.4- \\
35.9)\end{array}$ & 0.17 & $\begin{array}{l}32.1(26.8- \\
37.4)\end{array}$ & $\begin{array}{l}17.5(10.4- \\
24.7)\end{array}$ & $\begin{array}{l}< \\
0.001\end{array}$ \\
\hline $\begin{array}{l}\text { Total physical } \\
\text { activity }\end{array}$ & $\begin{array}{l}53.2(44.9- \\
61.4)\end{array}$ & $\begin{array}{l}43.5(19.7- \\
67.2)\end{array}$ & 0.44 & $\begin{array}{l}53.9(44.9- \\
62.8)\end{array}$ & $\begin{array}{l}27.3(13.2- \\
41.4)\end{array}$ & 0.002 \\
\hline $\begin{array}{l}\text { Sitting time } \\
\text { (hours/week) }\end{array}$ & $\begin{array}{l}34.6(32.8- \\
36.5)\end{array}$ & $\begin{array}{l}34.2(30.0- \\
38.8)\end{array}$ & 0.86 & $\begin{array}{l}34.3(32.6- \\
36.1)\end{array}$ & $\begin{array}{l}37.9(31.8- \\
44.8)\end{array}$ & 0.28 \\
\hline
\end{tabular}

Similarly, after adjusting for age, $\mathrm{BMI}$ and depression, we found no association between levels of pain intensity and participation in work-related activity $(\mathrm{OR}(95 \% \mathrm{Cl})=0.53(0.28-1.03), p=0.06)$, leisure activity $(0.96(0.53-1.73), p=0.89)$, active transportation $(1.50(0.80-2.81), p=0.21)$ or vigorous activity (1.02 (0.53-1.94), $p=0.96)$ (Table 4, model 1$)$. These results remained non-significant after further adjustment for working status (Table 4, model 2; Fig. 2). 
Table 4

The association between levels of low back pain intensity and disability and participation in physical activity.

\begin{tabular}{|c|c|c|c|c|c|c|c|c|}
\hline & Low be & k pain in & ensity & & Low be & k disabil & & \\
\hline & $\begin{array}{l}\text { No/ } \\
\text { low } \\
(n= \\
392)\end{array}$ & $\begin{array}{l}\text { High } \\
(n= \\
71)\end{array}$ & $\begin{array}{l}\text { OR }(95 \% \\
\mathrm{Cl})^{\mathrm{a}}\end{array}$ & $\begin{array}{l}\mathrm{P} \\
\text { value }\end{array}$ & $\begin{array}{l}\text { No/ } \\
\text { low } \\
(n= \\
425)\end{array}$ & $\begin{array}{l}\text { High } \\
(n= \\
38)\end{array}$ & $\begin{array}{l}\text { OR (95\% } \\
\text { Cl) })^{a}\end{array}$ & $\begin{array}{l}P \\
\text { value }\end{array}$ \\
\hline Model $1^{b}$ & & & & & & & & \\
\hline $\begin{array}{l}\text { Work-related } \\
\text { activity, n (\%) }\end{array}$ & $\begin{array}{l}142 \\
(36.6)\end{array}$ & $\begin{array}{l}16 \\
(23.5)\end{array}$ & $\begin{array}{l}0.53 \\
(0.28- \\
1.03)\end{array}$ & 0.06 & $\begin{array}{l}149 \\
(35.6)\end{array}$ & $\begin{array}{l}9 \\
(24.3)\end{array}$ & $\begin{array}{l}0.78 \\
(0.34- \\
1.81)\end{array}$ & 0.57 \\
\hline $\begin{array}{l}\text { Leisure activity, n } \\
(\%)\end{array}$ & $\begin{array}{l}268 \\
(69.1)\end{array}$ & $\begin{array}{l}43 \\
(63.2)\end{array}$ & $\begin{array}{l}0.96 \\
(0.53- \\
1.73)\end{array}$ & 0.89 & $\begin{array}{l}294 \\
(70.2)\end{array}$ & $\begin{array}{l}17 \\
(45.9)\end{array}$ & $\begin{array}{l}0.41 \\
(0.19- \\
0.85)\end{array}$ & 0.02 \\
\hline $\begin{array}{l}\text { Active } \\
\text { transportation, } \mathrm{n} \\
(\%)\end{array}$ & $\begin{array}{l}264 \\
(68.0)\end{array}$ & $\begin{array}{l}51 \\
(75.0)\end{array}$ & $\begin{array}{l}1.50 \\
(0.80- \\
2.81)\end{array}$ & 0.21 & $\begin{array}{l}291 \\
(69.5)\end{array}$ & $\begin{array}{l}24 \\
(64.9)\end{array}$ & $\begin{array}{l}0.95 \\
(0.44- \\
2.06)\end{array}$ & 0.89 \\
\hline $\begin{array}{l}\text { Vigorous activity, } \\
\text { n (\%) }\end{array}$ & $\begin{array}{l}120 \\
(30.9)\end{array}$ & $\begin{array}{l}17 \\
(25)\end{array}$ & $\begin{array}{l}1.02 \\
(0.53- \\
1.94)\end{array}$ & 0.96 & $\begin{array}{l}134 \\
(32.0)\end{array}$ & $\begin{array}{l}3 \\
(8.11)\end{array}$ & $\begin{array}{l}0.16 \\
(0.03- \\
0.70)\end{array}$ & 0.02 \\
\hline Model $2^{c}$ & & & & & & & & \\
\hline $\begin{array}{l}\text { Work-related } \\
\text { activity, n (\%) }\end{array}$ & $\begin{array}{l}142 \\
(36.6)\end{array}$ & $\begin{array}{l}16 \\
(23.5)\end{array}$ & $\begin{array}{l}0.70 \\
(0.33-1.5)\end{array}$ & 0.34 & $\begin{array}{l}149 \\
(35.6)\end{array}$ & $\begin{array}{l}9 \\
(24.3)\end{array}$ & $\begin{array}{l}1.38 \\
(0.49- \\
3.89)\end{array}$ & 0.54 \\
\hline $\begin{array}{l}\text { Leisure activity, } \mathrm{n} \\
(\%)\end{array}$ & $\begin{array}{l}268 \\
(69.1)\end{array}$ & $\begin{array}{l}43 \\
(63.2)\end{array}$ & $\begin{array}{l}1.00 \\
(0.55- \\
1.83)\end{array}$ & 0.99 & $\begin{array}{l}294 \\
(70.2)\end{array}$ & $\begin{array}{l}17 \\
(45.9)\end{array}$ & $\begin{array}{l}0.44 \\
(0.21- \\
0.94)\end{array}$ & 0.03 \\
\hline $\begin{array}{l}\text { Active } \\
\text { transportation, } \mathrm{n} \\
(\%)\end{array}$ & $\begin{array}{l}264 \\
(68.0)\end{array}$ & $\begin{array}{l}51 \\
(75.0)\end{array}$ & $\begin{array}{l}1.40 \\
(0.74- \\
2.65)\end{array}$ & 0.30 & $\begin{array}{l}291 \\
(69.5)\end{array}$ & $\begin{array}{l}24 \\
(64.9)\end{array}$ & $\begin{array}{l}0.89 \\
(0.41- \\
1.95)\end{array}$ & 0.78 \\
\hline $\begin{array}{l}\text { Vigorous activity, } \\
\mathrm{n}(\%)\end{array}$ & $\begin{array}{l}120 \\
(30.9)\end{array}$ & $\begin{array}{l}17 \\
(25)\end{array}$ & $\begin{array}{l}1.01 \\
(0.52- \\
1.97)\end{array}$ & 0.97 & $\begin{array}{l}134 \\
(32.0)\end{array}$ & $\begin{array}{l}3 \\
(8.11)\end{array}$ & $\begin{array}{l}0.17 \\
(0.04- \\
0.75)\end{array}$ & 0.02 \\
\hline
\end{tabular}

\section{Associations Between Low Back Disability And Physical Activity}


The associations between physical activity (MET; hours/week) and low back disability are shown in Table 3 and Fig. 1. After adjustment for age, BMI and depression (model 1), there was no significant difference in walking between women with high levels of back disability and those with no or low levels of back disability (MET (hours/week); estimated marginal median $(95 \% \mathrm{Cl})=7.6(2.7-12.5)$ vs. 11.4 (9.8$13.0), p=0.15)$, but less total $(28.4(14.5-42.3)$ vs. $54.4(45.3-63.6), p=0.001)$ and moderate physical activity (19.5(11.3-28.8) vs. $32.1(26.5-37.8), p=0.01)$. Moreover, sitting time per week was not significantly different between women with high levels of disability and those with no or low levels of disability (estimated marginal medians $(95 \% \mathrm{Cl})=37.2(31.1-43.8)$ vs. $34.5(32.7-36.2), p=0.41)$. After an additional adjustment for working status in model 2, we found that women with high levels of back disability also performed less domestic and garden activities compared to those with no or low levels of disability (MET (hours/week): 14.4(7.2-21.7) vs. 23.5(19.0-28.0), $p=0.02$ ).

After adjusting for age, BMI and depression (Table 4, model 1), we found that women with high levels of back disability were less likely to participate in leisure and vigorous activity $(\mathrm{OR}(95 \% \mathrm{Cl})=0.41(0.19-$ $0.85), p=0.02$ and $0.16(0.03-0.70), p=0.02$ respectively) compared to women with no or low levels of disability. There were no significant association between levels of disability and participation in workrelated $(0.78(0.34-1.81), p=0.57)$ and active transportation activities $(0.95(0.44-2.06), p=0.89)$. With further adjustment for working status, the results remained unchanged (Table 4, model 2; Fig. 2).

\section{Discussion}

In this study we found that community-based women with high levels of back disability, not back pain intensity, performed less overall physical activity compared to those with no or low levels of back pain and disability. Moreover, those with high levels of back disability differed in the intensities and types of physical activity they reported, performing less moderate and vigorous activities, as well as less discretionary physical activity, including leisure, domestic and gardening activities. These findings not only identify an important subgroup of individuals in the community that have high levels of back disability in combination with reduced physical activity, but also highlight the need for further investigation to examine the bi-directional nature of this relationship and identify potential targets for increasing physical activity and reducing disabling LBP.

We found that women with high levels of back disability performed only $50 \%$ of the physical activity of those with no or low levels of back disability. In addition, only $46 \%$ of the women with high disability performed leisure activity and as few as $8 \%$ were involved with vigorous physical activity. The amount of moderate and vigorous physical activity reported by these women was lower than that recommended by the World Health Organisation Guidelines, which suggest that individuals should perform 20 METS (hours/week) of moderate or vigorous physical activity per week, or an equivalent combination to gain health benefits (15). While it may be unrealistic for some women with high back disability to achieve these recommended activity levels, it is important to recognise that this may have major implications for their general health, including the development and management of co-morbidities, such as obesity and psychological conditions. Our data support this theory, as the women in our cohort with high disability 
had a mean BMI of $30 \mathrm{~kg} / \mathrm{m}^{2}$ with $50 \%$ classified as obese, and almost $16 \%$ reported depression. Understanding this burden of low back disability with regard to physical activity levels is important, given low back disability is the leading cause of disability globally (1), the majority of the socioeconomic burden associated with LBP results from those with high levels of disability (16), and obesity is in epidemic proportions $(17,18)$. Overall, these findings highlight the poor health and lack of daily physical activity performed by community-based women with high back disability.

In contrast to disability, we found no differences in physical activity in those with different levels of back pain. These results are novel, as previous systematic reviews and epidemiological studies have largely examined whether physical activity is a risk factor for LBP, rather than characterising the physical activity performed by individuals with different levels of pain and disability $(5,6,19)$. Moreover, few epidemiological studies have examined the relationship between both LBP and disability and physical activity in community-based individuals (7). The current findings suggest that an individual's physical activity is not linked to their level of pain, but rather to their level of function. This may be explained by current theoretical models, such as the Fear-avoidance model, which proposes that if an individual believes that their LBP is an indicator of severe tissue damage and certain activities will cause reinjury, they will display avoidance behaviours that will ultimately result in less physical activity and high levels of disability $(20,21)$. Conversely, individuals who believe that they do not have a serious condition and movement will assist their recovery, will move and be physically active despite their pain levels $(20,22-$ 24). Overall, the current results highlight the importance of targeting individuals with high levels of disability, rather than high levels of pain, when improving physical activity levels for LBP.

This study also found that individuals with high levels of disability differed in both the intensities and types of physical activity they performed compared to those with no or low levels of disability. Women who were highly disabled by their back pain reported less involvement in moderate and vigorous activity, as well as discretionary activities, including leisure, domestic and garden activities. These findings have significant implications. Given there is growing evidence that moderate intensity exercise improves pain sensitivity for musculoskeletal pain (25), our findings suggest that individuals with high disability may not be receiving the potential benefits in pain relief associated with this intensity of activity. Individuals who are not participating in recommended levels of moderate or vigorous exercise will also not receive the important health benefits of exercise, from improved mood and mental health, weight regulation and increased energy levels. Furthermore, the women who were undertaking reduced levels of discretional activities, including leisure, domestic and gardening activities, may experience a reduction in their psychosocial wellbeing as a result of not being able to fulfill their familial roles, which in turn may further impact on their back disability. While further longitudinal investigation is needed, these findings suggest that there is a need to focus on specific intensities and types of physical activity to improve the physical activity levels of individuals with high levels of disability.

There are some limitations in our study. Due to the cross-sectional design, a causal relationship between low back disability and physical activity could not be determined. While we had a modest sample size with a relatively small number of participants with high intensity LBP and disability, it was comparable to 
the prevalence of LBP and disability in the Australian population (26). Our study participants were recruited from a parent study of community-based women, who were originally recruited through random sampling from the state electoral roll, and without having LBP as a recruitment criterion. Our sample had comparable baseline demographics to those of the parent study (age mean (SD): 50 (14.4); BMI mean (SD): $\left.27.8(6.5) \mathrm{kg} / \mathrm{m}^{2}\right)(10)$. While the IPAQ is a self-reported questionnaire, which makes it susceptible to recall bias and participants may over-report physical activity (27), it has been shown to have good reliability and validity when compared to objective physical activity measures (13).

There are a number of strengths to our study. We used validated questionnaires to measure LBP intensity and disability. Our data allowed us to examine different levels of pain and disability (ie no/low versus high) and to investigate both participation and METs for different types and intensities of physical activity. Given we recruited our community-based women from the Victorian state electoral roll and without reference to the presence of back pain, it suggests that our results have high generalizability to the general community.

\section{Conclusions}

Our study found that high levels of low back disability, not back pain intensity, were associated with reduced physical activity, including less total activity, moderate and vigorous activities and discretionary types of activity. These findings highlight the excessive burden of high levels of back disability with respect to physical activity and the specific subgroups of individuals with low back disability who participate in less physical activity. While further investigation is needed to examine this likely bidirectional relationship, these data highlight the potential amounts, intensity and types of physical activity that need to be investigated further to increase physical activity and reduce disability in community-based women with LBP.

\section{Abbreviations}

LBP

low back pain

CPG

The Chronic Pain Grade questionnaire

IPAQ

The International Physical Activity Questionnaire

MET

Metabolic Equivalent Task

$\mathrm{BMI}$

Body Mass Index

$\mathrm{Cl}$

Confidence Intervals

SD 


\section{Declarations}

\section{Ethics approval and consent to participate}

Written informed consent was obtained from all participants and the research was approved by Monash University Human Research and Ethics Committee (CF07/2961-2006000113).

\section{Consent for publication}

Not applicable.

\section{Availability of data and materials}

Data will be shared upon reasonable request and approval by Assoc Prof. Donna Urquhart (Donna.Urquhart@monash.edu)

\section{Competing interests}

None declared

\section{Source of funding}

This study was funded by a Monash University Strategic Grant and Physiotherapy Research Foundation Grant (Continence and Women's Health). BA was supported by a scholarship from Princess Nourah Bint Abdulrahman University (Riyadh, Saudi Arabia). YW is the recipient of National Health and Medical Research Council Translating Research into Practice Fellowship (APP1168185). DU was supported by a National Health and Medical Research Council Career Development Fellowship (Level 2 \#1142809). For the remaining authors, none were declared.

\section{Authorship contribution}

Authors have contributed to the study as follows; study conception and design (BA, DMU and FMC), analysis of the data (BA, DMU and $\mathrm{SH}$ ), interpretation of data (BA, DMU, SH, YW and ZK), drafting of the manuscript (BA, DMU), critical review of the manuscript (BA, DMU, FMC, SH, YW and ZK). All authors discussed the results, commented and approved the final version of the manuscript to be published.

\section{Acknowledgements}

Not applicable

\section{References}


1. Hoy D, March L, Brooks P, Blyth F, Woolf A, Bain C, et al. The global burden of low back pain: estimates from the Global Burden of Disease 2010 study. Ann Rheum Dis. 2014;73(6):968-74.

2. Krismer M, van Tulder M. Low back pain (non-specific). Best Pract Res Clin Rheumatol. 2007;21(1):77-91.

3. Oliveira CB, Maher CG, Pinto RZ, Traeger AC, Lin C-WC, Chenot J-F, et al. Clinical practice guidelines for the management of non-specific low back pain in primary care: an updated overview. Eur Spine J. 2018;27(11):2791-803.

4. Hartvigsen J. hancock MJ, kongsTed a, louW Q, ferreira Ml, genevay s, hoy d, karPPinen J, Pransky g, siePer J, sMeeTs rJ, underWood M; Lancet Low Back Pain Series Working Group. What low back pain is and why we need to pay attention. Lancet. 2018;391:2356-67.

5. Hendrick P, Milosavljevic S, Hale L, Hurley D, McDonough S, Ryan B, et al. The relationship between physical activity and low back pain outcomes: a systematic review of observational studies. Eur Spine J. 2011;20(3):464-74.

6. Oliveira CB, Pinheiro MB, Teixeira RJ, Franco MR, Silva FG, Hisamatsu TM, et al. Physical activity as a prognostic factor of pain intensity and disability in patients with low back pain: A systematic review. Eur J Pain. 2019;23(7):1251-63.

7. Lin CC-W, McAuley HJ, Macedo CL, Barnett JD, Smeets AR, Verbunt AJ. Relationship between physical activity and disability in low back pain: A systematic review and meta-analysis. Pain. 2011;152(3):607-13.

8. Ezeukwu AO, Ebisike G, John JN, Okezue O, John DO. Self-reported physical activity level and associated factors among patients with non-specific chronic low back pain. Journal of Rehabilitation Sciences Research. 2019;6(1):6-14.

9. Sokunbi G, Akindele M, Bello B, Bello U, Terso-ivase I. Physical activity pattern and its association with functional limitation, physical health and mental well being of nurses with low back pain. Scholar Reports. 2016;1(2):1-11.

10. Davison SL, Bell R, Donath S, Montalto JG, Davis SR. Androgen Levels in Adult Females: Changes with Age, Menopause, and Oophorectomy. The Journal of Clinical Endocrinology Metabolism. 2005;90(7):3847-53.

11. Von Korff JM, Ormel FJ, Keefe FF, Dworkin FS. Grading the severity of chronic pain. Pain. 1992;50(2):133-49.

12. Ainsworth EB, Haskell LW, Whitt CM, Irwin LM, Swartz MA, Strath JS, et al. Compendium of Physical Activities: an update of activity codes and MET intensities. Med Sci Sports Exerc. 2000;32(9 Suppl):498-516.

13. Craig LC, Marshall LA, Sjöström EM, Bauman LA, Booth EM, Ainsworth FB, et al. International Physical Activity Questionnaire: 12-Country Reliability and Validity. Med Sci Sports Exerc. 2003;35(8):1381-95.

14. Sjostram M, Ainsworth B, Bauman A, Bull F, Craig C, Sallis J. Guidelines for data processing and analysis of the International Physical Activity Questionnaire (IPAQ) - short and long forms: Nov 2005. 
Available at wwwipaqkise/scoring Accessed. 2009;5(11).

15. Organization WH. Global Recommendations on Physical Activity for Health. Geneva2010.

16. Nachemson AL. Newest Knowledge of Low Back Pain A Critical Look. Clin Orthop Relat Res. 1992;\&NA(279):8-20.

17. Finucane MM, Stevens GA, Cowan MJ, Danaei G, Lin JK, Paciorek CJ, et al. National, regional, and global trends in body-mass index since 1980: systematic analysis of health examination surveys and epidemiological studies with 960 country-years and $9 \cdot 1$ million participants. The Lancet. 2011;377(9765):557-67.

18. Ng M, Fleming T, Robinson M, Thomson B, Graetz N, Margono C, et al. Global, regional, and national prevalence of overweight and obesity in children and adults during 1980-2013: a systematic analysis for the Global Burden of Disease Study 2013. The Lancet. 2014;384(9945):766-81.

19. Heneweer H, Staes F, Aufdemkampe G, Rijn M, Vanhees L. Physical activity and low back pain: a systematic review of recent literature. Eur Spine J. 2011;20(6):826-45.

20. Huijnen IPJ, Verbunt JA, Peters ML, Smeets RJEM, Kindermans HPJ, Roelofs J, et al. Differences in activity-related behaviour among patients with chronic low back pain. Eur J Pain. 2011;15(7):74855.

21. Plaas H, Sudhaus S, Willburger R, Hasenbring MI. Physical activity and low back pain: the role of subgroups based on the avoidance-endurance model. Disabil Rehabil. 2014;36(9):749-55.

22. Hasenbring Ml, Plaas $\mathrm{H}$, Fischbein $\mathrm{B}$, Willburger R. The relationship between activity and pain in patients 6 months after lumbar disc surgery: Do pain-related coping modes act as moderator variables? Eur J Pain. 2006;10(8):701-9.

23. Hasenbring MI, Hallner D, Rusu AC. Fear-avoidance- and endurance-related responses to pain: Development and validation of the Avoidance-Endurance Questionnaire (AEQ). Eur J Pain. 2009;13(6):620-8.

24. Scholich SL, Hallner D, Wittenberg RH, Hasenbring MI, Rusu AC. The relationship between pain, disability, quality of life and cognitive-behavioural factors in chronic back pain. Disabil Rehabil. 2012;34(23):1993-2000.

25. Naugle KM, Fillingim RB, Riley JL. A Meta-Analytic Review of the Hypoalgesic Effects of Exercise. J Pain. 2012;13(12):1139-50.

26. Walker BF, Muller R, Grant WD. Low Back Pain in Australian Adults. Prevalence and Associated Disability. J Manipulative Physiol Ther. 2004;27(4):238-44.

27. Dyrstad MS, Hansen HB, Holme MI, Anderssen AS. Comparison of Self-reported versus Accelerometer-Measured Physical Activity. Med Sci Sports Exerc. 2014;46(1):99-106.

\section{Figures}




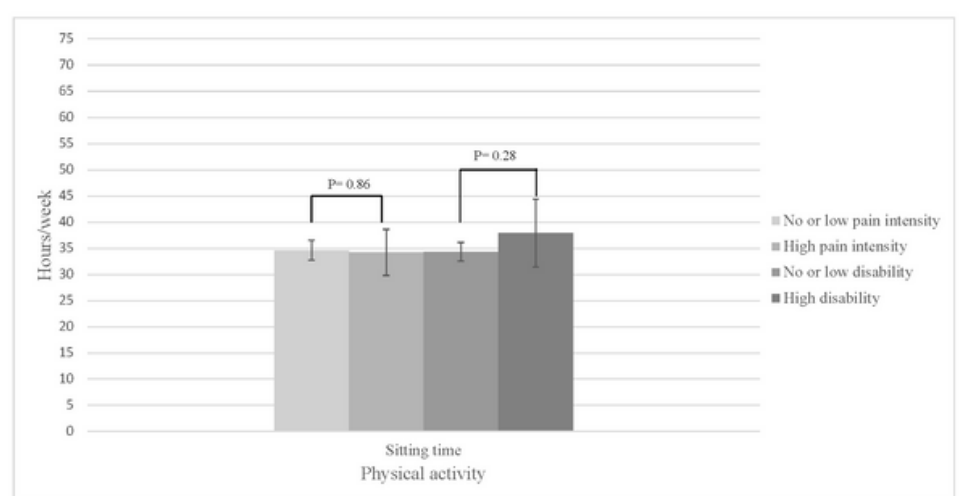

A

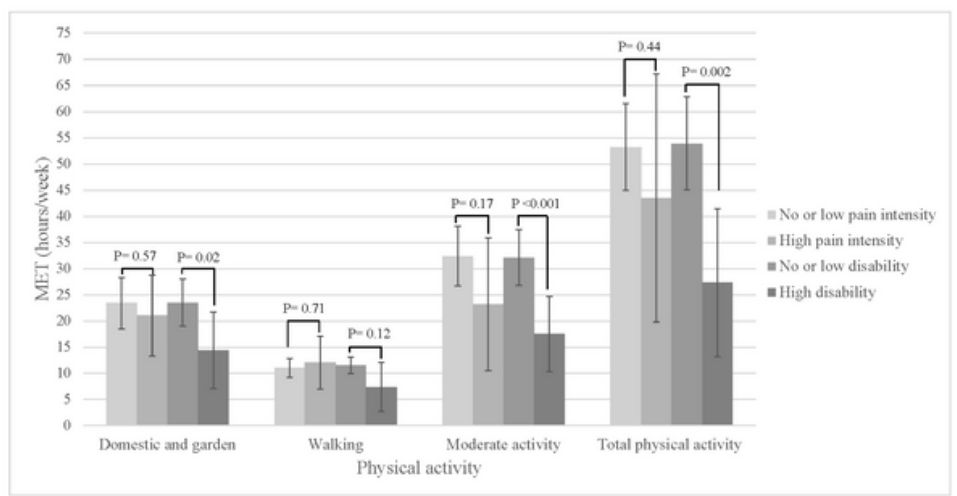

B

\section{Figure 1}

(A). Association between levels of low back pain intensity and disability (no/low versus high) and physical activity (MET (hours/week)) (adjusted marginal median, 95\% Cls), adjusted for age, BMI, depression and working status. (B). Association between levels of low back pain intensity and disability (no/low versus high) and sitting time (hours/week) (adjusted marginal medians, 95\% Cls), adjusted for age, BMI, depression and working status.

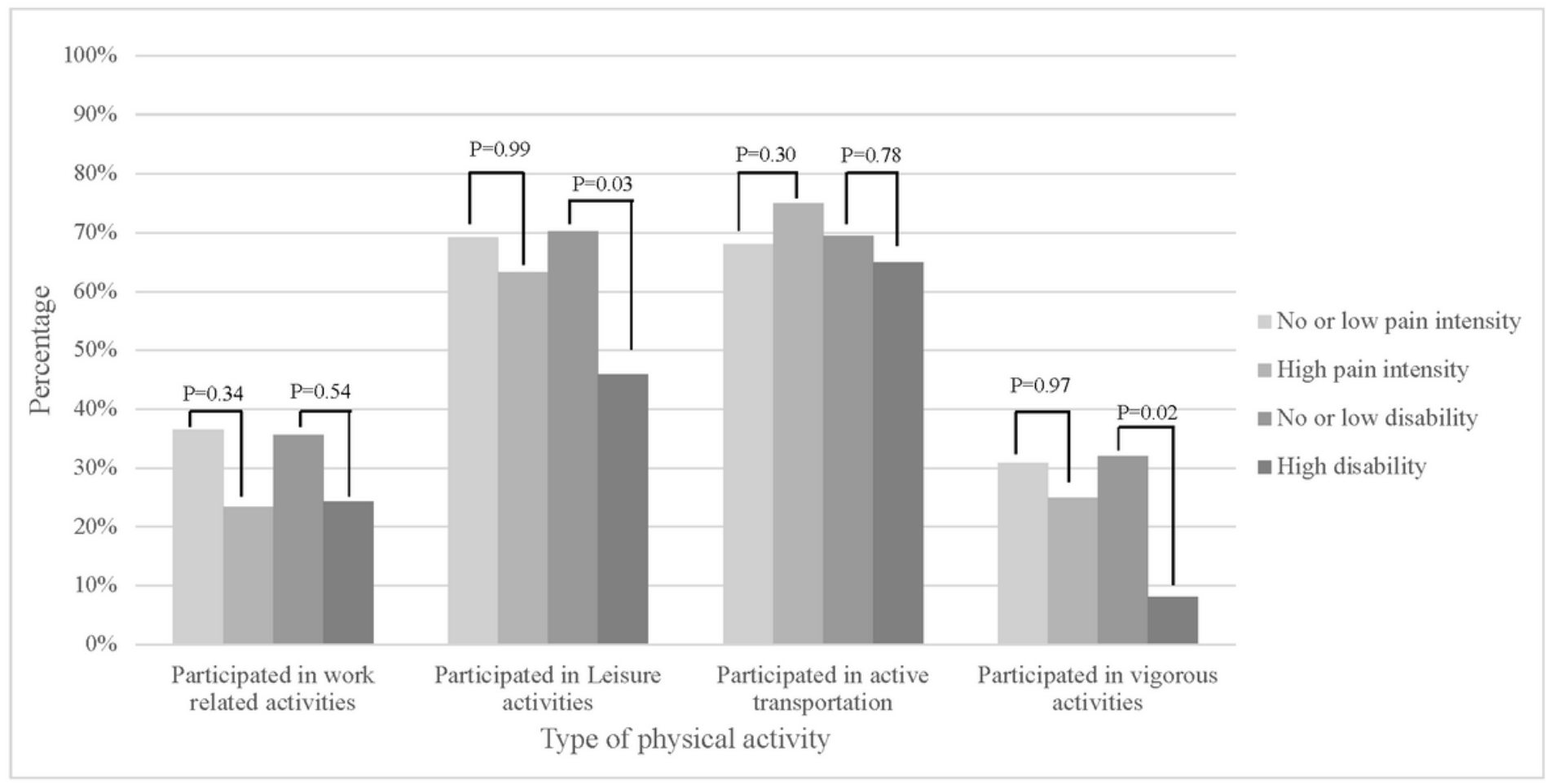

\section{Figure 2}

Association between levels of low back pain intensity and disability (no/low versus high) and participation in physical activity (\%), adjusted for age, BMI, depression and working status. 